Check for updates

Cite this: J. Mater. Chem. B, 2020, 8, 9566

Received 13th July 2020,

Accepted 20th September 2020

DOI: $10.1039 / \mathrm{dOtb01731k}$

rsc.li/materials-b

\title{
Rapid and cytocompatible cell-laden silk hydrogel formation via riboflavin-mediated crosslinking $\dagger$
}

\author{
Susanna Piluso, (D) abc Daniela Flores Gomez, ${ }^{\text {ab }}$ Inge Dokter, ${ }^{\text {ab }}$ \\ Liliana Moreira Texeira, ${ }^{c d}$ Yang Li, ${ }^{\text {ab }}$ Jeroen Leijten, ${ }^{c}$ René van Weeren, ${ }^{\text {bd }}$ \\ Tina Vermonden, (D) ${ }^{e}$ Marcel Karperien ${ }^{c}$ and Jos Malda*abd
}

\begin{abstract}
Bioactive hydrogels based on naturally-derived polymers are of great interest for regenerative medicine applications. Among naturally-derived polymers, silk fibroin has been extensively explored as a biomaterial for tissue engineering due to its unique mechanical properties. Here, we demonstrate the rapid gelation of cell-laden silk fibroin hydrogels by visible light-induced crosslinking using riboflavin as a photo-initiator, in presence of an electron acceptor. The gelation kinetics were monitored by in situ photo-rheometry. Gelation was achieved in minutes and could be tuned owing to its direct proportionality to the electron acceptor concentration. The concentration of the electron acceptor did not affect the elastic modulus of the hydrogels, which could be altered by varying the polymer content. Further, the biocompatible riboflavin photo-initiator combined with sodium persulfate allowed for the encapsulation of cells within silk fibroin hydrogels. To confirm the cytocompatibility of the silk fibroin formulations, three cell types (articular cartilage-derived progenitor cells, mesenchymal stem cells and dental-pulp-derived stem cells) were encapsulated within the hydrogels, which associated with a viability $>80 \%$ for all cell types. These results demonstrated that fast gelation of silk fibroin can be achieved by combining it with riboflavin and electron acceptors, which results in a hydrogel that can be used in tissue engineering and cell delivery applications.
\end{abstract}

\section{Introduction}

Cells in tissues and organs are surrounded by extracellular matrix (ECM), a complex and highly organized bioactive structure that provides mechanical support and guides cell adhesion, proliferation and differentiation. ${ }^{1,2}$ Hydrogels are threedimensional (3D) water-swollen polymer networks and ideal candidates to mimic the intricate and organized network of the native ECM, due to their high water content, viscoelasticity, and biocompatibility. ${ }^{3,4}$ Importantly, polymers can be molecularly engineered to make hydrogels biodegradable, and their mechanical and physical properties can be tuned to mimic those of native tissues. ${ }^{5}$ Hydrogels for tissue engineering can be

\footnotetext{
${ }^{a}$ Department of Orthopaedics, University Medical Center Utrecht, Utrecht University, Utrecht, The Netherlands. E-mail: j.malda@umcutrecht.nl

${ }^{b}$ Regenerative Medicine Utrecht, Utrecht University, Utrecht, The Netherlands

${ }^{c}$ Department of Developmental BioEngineering, Technical Medical Centre,

University of Twente, Enschede, The Netherlands

${ }^{d}$ Department of Clinical Sciences, Faculty of Veterinary Medicine, Utrecht University, Utrecht, The Netherlands

${ }^{e}$ Department of Pharmaceutics, Utrecht Institute for Pharmaceutical Sciences (UIPS), Science for Life, Utrecht University, Universiteitsweg 99, 3508 TB, Utrecht, The Netherlands

$\dagger$ Electronic supplementary information (ESI) available. See DOI: 10.1039/d0tb01731k
}

made from synthetic or natural polymers, with the latter being of most interest since they possess inherent biological cues that promote interactions with cells. Examples of natural derived polymers include ECM-derived components, such as collagen, fibrin, hyaluronic acid, or polymers derived from other biological sources, such as silk fibroin. ${ }^{1,5}$ Silk fibroin is a fibrous protein that has been extensively exploited for biomedical applications due to its biocompatibility, excellent mechanical properties and biodegradation. ${ }^{6}$ Applications, include corneal wound healing, ${ }^{7,8}$ and tissue engineering of skin, ${ }^{9}$ cartilage, ${ }^{10}$ and bone tissue. ${ }^{11}$ The unique mechanical properties of silk are due to its particular molecular conformation consisting of $\beta$-sheet domains within a network of semi-amorphous regions. ${ }^{11}$ The semi-crystalline regions convey the mechanical strength, whereas the amorphous regions provide elasticity. Silk fibroin derived from Bombyx mori consists of light (26 kDa) and heavy chains (391 kDa), with the latter containing 11 short hydrophilic blocks (consisting of approximately 31 amino acids) and 12 hydrophobic blocks. ${ }^{12}$ This particular arrangement confers a block copolymer-like molecular structure to silk, and is responsible for the unique mechanical properties. The hydrophobic blocks contain mainly glycine-X (G-X) motifs, were $\mathrm{X}$ can be alanine $(\mathrm{A} ; 65 \%)$, serine $(\mathrm{S} ; 23 \%)$ or tyrosine (about $9 \%$ ). ${ }^{12}$ The formation of $\beta$-sheet domains is due to the 
highly repetitive sequence GAGAGS and can be triggered by various processing techniques including sonication, vortexing, lowering the $\mathrm{pH}$, and methanol treatment. ${ }^{11}$ Although these techniques result in the formation of films and porous scaffolds with excellent mechanical properties, most of them are not suitable for the preparation of cell-laden hydrogels, for which mild reaction conditions are required. Silk fibroin hydrogels have been prepared also via chemical crosslinking, for example with genipin, ${ }^{13}$ epichlorohydrin ${ }^{14}$ and via enzymatic crosslinking. ${ }^{6}$ For instance, biocompatible silk fibroin hydrogels have been obtained by using tyrosinase ${ }^{15}$ or horseradish peroxidase (HRP) and hydrogen peroxide $\left(\mathrm{H}_{2} \mathrm{O}_{2}\right)$ as initiator (which induce network formation via di-tyrosine crosslinks). ${ }^{16}$ In this case, a faster gelation could be achieved by increasing either HRP or $\mathrm{H}_{2} \mathrm{O}_{2}$ concentrations, but this may entail a potential immune response or induce toxicity in vivo. ${ }^{17}$ An alternative approach is the functionalization of silk fibroin with tyramine to increase the availability of phenol moieties for crosslinking. ${ }^{6}$ Although this strategy improves the gelation kinetics, this crosslinking method does not enable spatial and temporal control. Opposingly, visible light-triggered reactions are advantageous in presence of living cells, since cells contains few visible light chromophores, but several molecules that absorb UV light ${ }^{18}$ and can be easy tuned for spatial-temporal sensitive applications. Previous reports have shown the visible lightinduced crosslinking of silk fibroin hydrogels using ruthenium as photo-initiator. ${ }^{19,20}$ The biocompatibility of ruthenium is unknown and under certain conditions might be highly toxic, ${ }^{21,22}$ therefore a photo-initiator of known biocompatibility is needed. Riboflavin (RB), also known as vitamin B2, is a non-toxic photoinitiator recently used for the preparation of silk fibroin hydrogels upon exposure to visible light. ${ }^{7}$ A main limitation for the use of RB, however, is the slow gelation kinetics, ${ }^{7,23,24}$ which would make this RB-based crosslinking not suitable for the encapsulation of cells within the hydrogel matrix. Here, we propose a method to achieve a fast and cytocompatible gelation of silk fibroin via RB-mediated crosslinking. Furthermore, we determine the cytocompatible sodium persulfate (SPS) concentration, that enables for safe rapid crosslinking in combination with RB. Such faster gelation times would result in more homogeneous cell distribution and would allow application of the material as a potential ink for the fabrication of complex three-dimensional (3D) structures via e.g. bioprinting technologies. We first evaluated the influence of SPS and RB on the gelation time, mechanical properties and swelling behaviour of silk fibroin hydrogels. Next, we investigated the cytocompatibility of RB-mediated crosslinking using three cell types, namely articular cartilage-derived progenitor cells (ACPCs), mesenchymal stem cells (MSCs) and dental pulp derived stem cells (DPSCs).

\section{Materials and methods}

\subsection{Preparation of aqueous silk fibroin solutions}

Aqueous silk fibroin solutions were prepared as previously described. ${ }^{25}$ Briefly, Bombyx mori silkworm cocoons (wildfibres, UK) were boiled in $0.02 \mathrm{M}$ sodium carbonate $\left(\mathrm{Na}_{2} \mathrm{CO}_{3}\right.$, Sigma-Aldrich) for $30 \mathrm{~min}$. The degummed fibers were dissolved at $20 \%(\mathrm{w} / \mathrm{v})$ in a $9.3 \mathrm{M}$ lithium bromide (LiBr, Acros Organics) solution at $70{ }^{\circ} \mathrm{C}$ for $1 \mathrm{~h}$, followed by dialysis against water for $48 \mathrm{~h}$, using cellulose dialyzing tubing (MWCO $3.5 \mathrm{kDa}$, Sigma Aldrich). The resulting $6 \%(\mathrm{w} / \mathrm{v})$ silk fibroin solution was concentrated to $12 \%$ (w/v) by dialysis against PEG (10 kDa, Sigma Aldrich).

\subsection{Rheology}

The gelation of silk fibroin solutions was monitored by in situ photo-rheometry using a Rheometer (Discovery HR-2, TA instruments, New Castle, DE, USA) fitted with a parallel plate of $20 \mathrm{~mm}$ diameter and a gap distance of $0.5 \mathrm{~mm}$, and equipped with a light curing system (600 lumen white lamp; 400-700 nm). To initiate gelation, the precursor solutions were placed on a quartz plate in a curing unit connected to visible light. The solutions were exposed to visible light $30 \mathrm{~s}$ after the start of each measurement for $25 \mathrm{~min}$. The elastic $\left(G^{\prime}\right)$ and viscous $\left(G^{\prime \prime}\right)$ moduli were recorded as function of time at room temperature at a frequency of $0.1 \mathrm{~Hz}$ and $1 \%$ strain. The solutions were prepared with silk fibroin at $6-8-10 \%(\mathrm{w} / \mathrm{v})$ mixed with different $\mathrm{RB} / \mathrm{SPS}$ ratios $(2 / 10 ; 2 / 20 ; 2 / 40)$ in water.

The viscoelastic properties of the resulting hydrogels were determined by conducting dynamic frequency sweeps (1-100 $\mathrm{rad} \mathrm{s}^{-1} ; 1 \%$ strain). All measurements were performed in the linear viscoelastic region. Finally, shear recovery experiments were performed to study the material response to application and removal of strain by performing time sweep measurements with alternating high (500\%) and low (100\%) strain.

The determined storage modulus was used to estimate the mesh size $(\xi, \mathrm{nm})$ of the hydrogels, which defines the distance between the crosslinking points. The mesh size was calculated using the equation below based on the rubber elasticity theory: ${ }^{6,26}$

$$
\xi=\left(\frac{G^{\prime} N_{\mathrm{A}}}{R T}\right)^{-\frac{1}{3}}
$$

Where $G^{\prime}$ is the storage modulus, $N_{\mathrm{A}}$ is the Avogadro constant, $R$ is the molar gas constant and $T$ is the temperature. The theory is based on the assumption that the hydrogel is purely elastic and neglects any physical interactions between the polymer chains.

The crosslinking density $\left(n_{\mathrm{e}}, \mathrm{mol} \mathrm{m}{ }^{-3}\right)$ of the hydrogels was calculated as follows: ${ }^{27}$

$$
n_{\mathrm{e}}=\left(\frac{G}{R T}\right)
$$

\subsection{Hydrogel formation}

Stock solution of RB (40 mM) and SPS (540 mM) were prepared in ultrapure water. The stock solution of RB was protected from light by covering the vial with aluminum foil. Silk fibroin was concentrated to $12 \%(\mathrm{w} / \mathrm{w})$ by dialysis against PEG. Hydrogel precursor solutions were diluted to the desired concentration, and the resulting mixture was pipetted into a PDMS mold containing voids of $8 \mathrm{~mm} \times 1 \mathrm{~mm}(50 \mu \mathrm{L})$ and exposed to visible light (600 lumen white lamp) for $25 \mathrm{~min}$. After synthesis, silk fibroin hydrogels were rinsed at least 4 times to wash out 


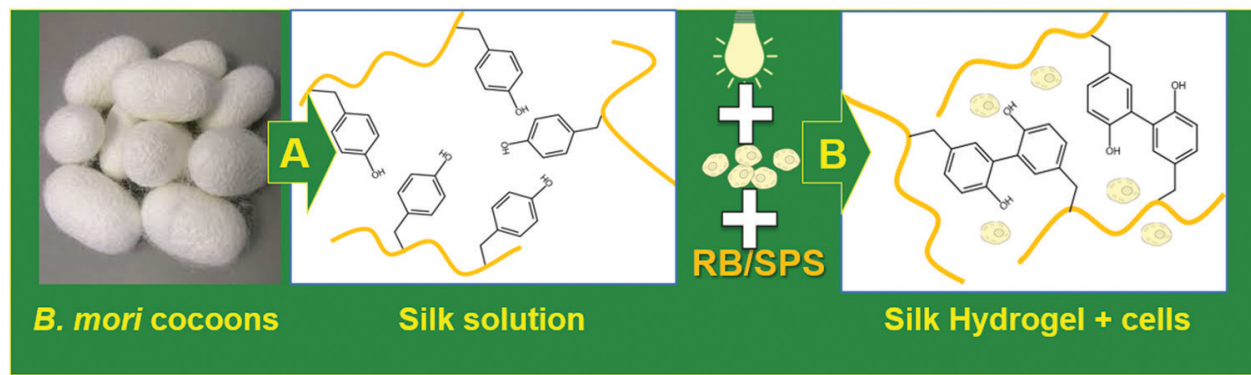

Fig. 1 Schematic of the fabrication of cell-laden silk fibroin hydrogels (A). In a first step (A), silk fibroin aqueous solutions extracted from cocoons are mixed with cells, followed by riboflavin (RB) and sodium persulfate (SPS). Exposure to visible light promote hydrogel formation through di-tyrosine crosslinks (B).

Table 1 Composition of cell laden silk fibroin hydrogels

\begin{tabular}{lllll}
\hline Sample ID $^{a}$ & Silk \% (w/v) & RB (mM) & SPS (mM) & Time (min) \\
\hline Silk_6-20 & 6 & 2 & 20 & 25 \\
Silk_8-10 & 8 & 2 & 10 & 25 \\
Silk_8-20 & 8 & 2 & 20 & 25 \\
Silk_8-40 & 8 & 2 & 40 & 25 \\
Silk_8-20(0.5) & 8 & 0.5 & 20 & 25 \\
Silk_8-20(5) & 8 & 5 & 20 & 25 \\
Silk_10-20 & 10 & 2 & 20 & 25
\end{tabular}

${ }^{a}$ Silk_ $x-y(z): x$ stands for silk fibroin $\% ; y$ for SPS concentration; $z$ stands for RB concentration if different than $2 \mathrm{mM}$.

any left RB and SPS. The strategy for the preparation of silk fibroin hydrogel formation is depicted in Fig. 1. Several hydrogel compositions were prepared by varying the concentration of SPS, RB and silk fibroin (as reported in Table 1).

\subsection{Swelling and sol fraction}

After synthesis, silk hydrogels were lyophilized, and their dried weight $\left(m_{\mathrm{d} 1}\right)$ was measured. The hydrogels were then incubated in $1 \mathrm{~mL}$ water at $37{ }^{\circ} \mathrm{C}$. After 24 hours, the swollen weight $\left(m_{\mathrm{s}}\right)$ of the samples was recorded. After swelling, the samples were lyophilized to obtain the final dry weight $\left(m_{\mathrm{d} 2}\right)$. The swelling ratio was determined as $\left(m_{\mathrm{s}}-m_{\mathrm{d} 2}\right) / m_{\mathrm{d} 2}$. The sol fraction, which is the mass fraction of not crosslinked polymer in the hydrogel network, was determined as $\left(m_{\mathrm{d} 1}-m_{\mathrm{d} 2}\right) / m_{\mathrm{d} 1} \times 100$.

\subsection{Evaluation of cellular viability following exposure to RB and SPS}

Equine ACPCs were isolated in our laboratory as previously described. ${ }^{28}$ ACPCs were expanded in DMEM, supplemented with $10 \% \mathrm{v} / \mathrm{v}$ fetal calf serum (FCS), $0.2 \mathrm{mM}$ ascorbic acid-2phosphate, $1 \%$ penicillin-streptomycin, $5 \mathrm{ng} \mathrm{mL}^{-1}$ basic fibroblast growth factor (bFGF) and 1\% non-essential amino acids solution in a humidified incubator at $37{ }^{\circ} \mathrm{C}$ with $5 \% \mathrm{CO}_{2}$. Cells at passage 4 were seeded onto 24 well plates at a density of $50 \times 10^{4}$ cells per $\mathrm{mL}$ and incubated overnight. At day 1 , seeding media was replaced with regular growth media supplemented with $\mathrm{RB}$ to reach a final concentration of $0.5 \mathrm{mM}$, $2 \mathrm{mM}$, and $5 \mathrm{mM}$ and with SPS at a final concentration of $5 \mathrm{mM}, 10 \mathrm{mM}$, and $20 \mathrm{mM}$. At $15 \mathrm{~min}, 1 \mathrm{~h}, 3 \mathrm{~h}$, and $24 \mathrm{~h}$ the well plates were removed from the incubator and viability was assessed by live/dead assay, and images were taken with an inverted microscope (Olympus BX51, Olympus, Germany). The live and dead cell numbers were determined with Image (NIH, USA) and viability was determined as the number of live cells divided by the total number of cells.

\subsection{Evaluation of SPS released form the hydrogels}

The amount of SPS released from the hydrogels was measured using a peroxide assay. Briefly, hydrogels (50 $\mu \mathrm{L}$ in volume) after preparation were swollen in $200 \mu \mathrm{L}$ of water, and every 15 min the swelling medium was collected and replaced with fresh one. The collected medium was then mixed at a 1:10 ratio with a working reagent consisting of a mixture of $25 \mathrm{mM}$ ammonium iron(II) sulfate/2.5 $\mathrm{M}$ sulfuric acid, and $100 \mathrm{mM}$ sorbitol/125 $\mu \mathrm{M}$ xylenol orange in water. The mixtures were incubated for $15 \mathrm{~min}$ at room temperature. The absorbance was measured at $595 \mathrm{~nm}$ and the concentration determined using a calibration curve based on hydrogen peroxide.

\subsection{Cell culture in silk fibroin hydrogels}

Prior to cell encapsulation, silk fibroin at $7 \%(\mathrm{w} / \mathrm{v})$, stock solutions of RB (40 mM) and SPS (540 mM) were sterile filtered. Human MSC and goat DPSC were expanded in $\alpha$ MEM supplemented with 10\% FCS, $0.2 \mathrm{mM}$ ascorbic acid-2-phosphate, $1 \%$ penicillin-streptomycin, and $1 \mathrm{ng} \mathrm{mL}^{-1}$ bFGF. MSCs, DPSCs and ACPCs were harvested using trypsin and mixed with silk fibroin to reach a final concentration of silk fibroin of $6 \%$ and a final cell density of $15 \times 10^{6}$ cells per mL. RB and SPS were added at a final concentration of $2 \mathrm{mM}$ and $20 \mathrm{mM}$, respectively. The hydrogel precursor mixture $(50 \mu \mathrm{L})$ was pipetted into a PDMS mold and exposed to visible light for $10 \mathrm{~min}$. After synthesis, silk fibroin hydrogels encapsulating MSC and DPSCs were rinsed at least 4 times with culture media and cultured for 7 days; silk fibroin-ACPCs hydrogels were rinsed and cultured for 28 days in chondrogenic medium (DMEM supplemented with $1 \% \mathrm{v} / \mathrm{v}$ insulin-transferrin selenium (ITS+ Premix, Corning, USA)), $0.2 \mathrm{mM}$ ascorbic acid-2-phosphate, 100 units per $\mathrm{mL}$ penicillin with $100 \mathrm{mg} \mathrm{mL}{ }^{-1}$ streptomycin (Life Technologies, The Netherlands), $100 \mathrm{nM}$ dexamethasone and $10 \mathrm{ng} \mathrm{mL}^{-1}$ transforming growth factor-b1 (TGF-b1). The media was refreshed every 2 days. 


\subsection{Evaluation of viability of cell-laden silk fibroin hydrogels}

Cell viability was evaluated at 1 and 7 days of culture for MSCs and DPSCs, and at 1 and 28 days of culture for ACPCs with a live/dead assay. At each time point the hydrogels were rinsed with PBS and incubated with calcein AM $(2 \mu \mathrm{M})$ and ethidium homodimer-1 $(4 \mu \mathrm{M})$ in PBS for $30 \mathrm{~min}$. The gels were then rinsed with PBS and imaged using confocal microscopy (Leica inverted microscope) at $10 \times$ magnification (green channel for calcein: $\lambda_{\mathrm{ex}}=488 \mathrm{~nm}$ and $\lambda_{\mathrm{em}}=525 \mathrm{~nm}$; red channel for ethidium homodimer: $\lambda_{\mathrm{ex}}=543 \mathrm{~nm}$ and $\lambda_{\mathrm{em}}=590 \mathrm{~nm}$ ). For each hydrogel, three z-stacks were taken, and the maximum intensity projection images were used for analysis. The viability was quantified as described in Section 2.4.

\subsection{Statistical analysis}

Data are represented as mean \pm standard deviation. Statistical comparisons were performed using the one-way (or two-way when necessary) analysis of variance (ANOVA) with GraphPad Prism 8 software. A value of $p<0.05$ was considered statistically significant. After ANOVA, the Tukey's method was used to test all pairwise mean comparisons.

\section{Results and discussion}

\subsection{Effect of SPS and silk fibroin content on gelation time}

To evaluate the effect of SPS and silk fibroin concentration on the gelation time, hydrogel formation was monitored by in situ photo-rheometry (Fig. 2). Hydrogels precursor solutions were placed between the rheometer plates and exposed to visible light. We first investigated the effect of SPS concentration by keeping the silk fibroin concentration constant at $8 \%(\mathrm{w} / \mathrm{v})$ and $\mathrm{RB}$ at $2 \mathrm{mM}$ (Fig. 2A(i)). Upon exposure to light, the elastic modulus $G^{\prime}$ started to increase up to a certain point till it reached a plateau. The point at which $G^{\prime}$ crosses over $G^{\prime \prime}$ is
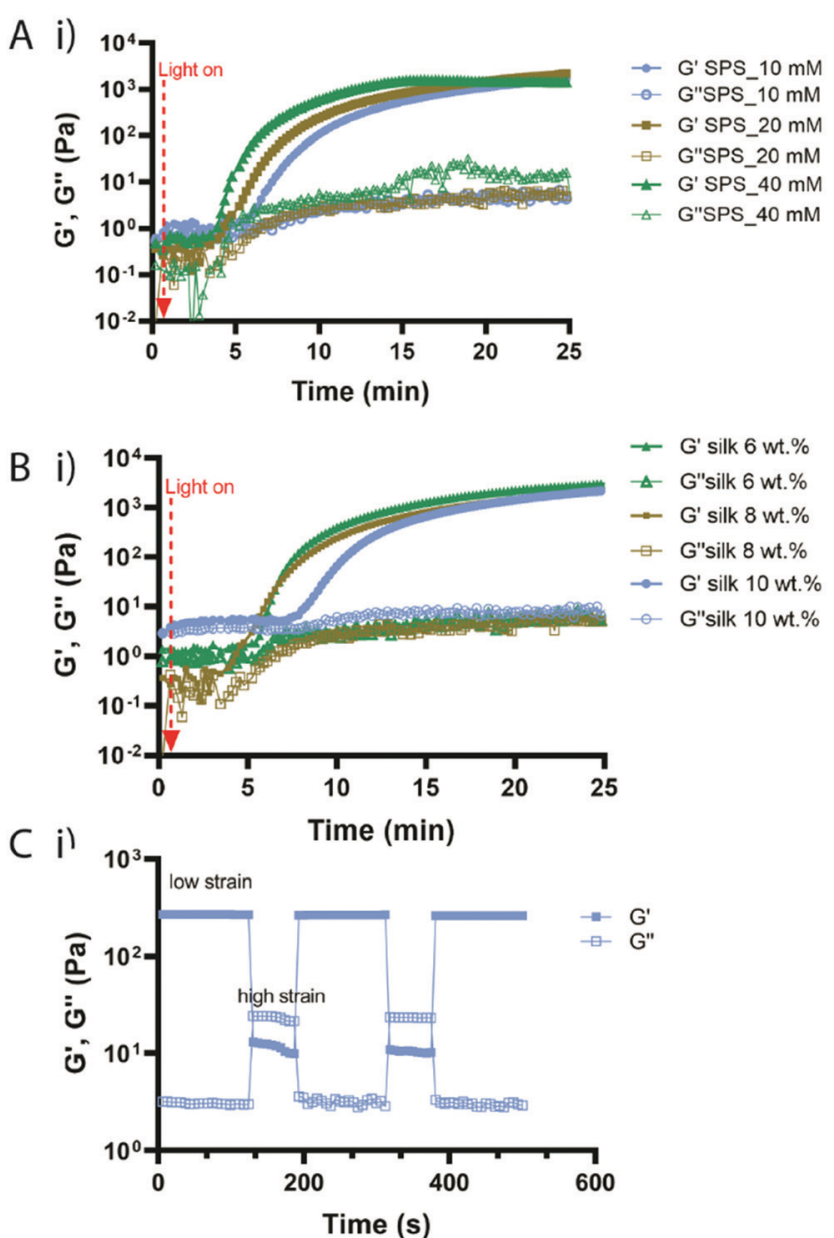

ii)

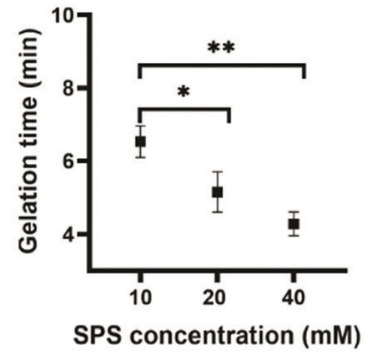

ii)
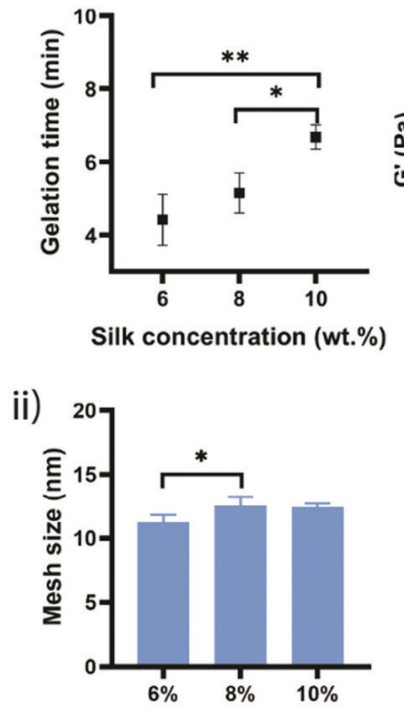

Silk concentration (wt.\%) iii)

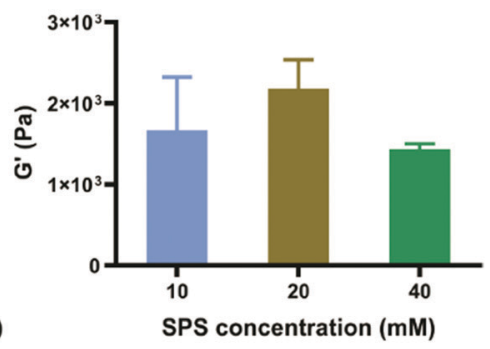

iii)

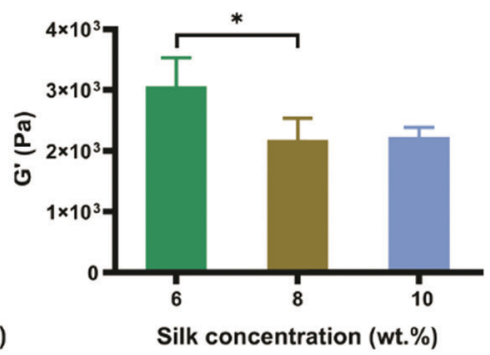

iii)

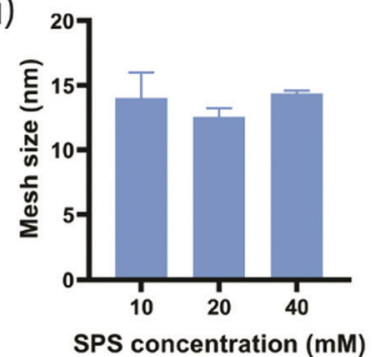

Fig. 2 Effect of SPS and silk fibroin content on the reaction kinetics of RB-mediated crosslinking. (A) (i) Rheological time sweep showing gelation kinetics as function of SPS concentration, upon exposure to visible light. The control without SPS has not been included because no hydrogel formation was observed in 25 min. Silk fibroin content was $6 \%$ and RB 2 mM; (ii) gelation time determined as the point at which $G^{\prime}$ crosses over $G^{\prime \prime}$; (iii) shear elastic modulus of silk fibroin hydrogel at $t=25 \mathrm{~min}$. (B) (i) Gelation kinetics as function of silk fibroin concentration, upon exposure to visible light. SPS concentration was $20 \mathrm{mM}$ and RB 2 mM; (ii) gelation time determined as the point at which $G^{\prime}$ crosses over G"; (iii) shear elastic modulus of silk fibroin hydrogel at $t=25 \mathrm{~min}$. (C) Cyclic oscillatory test of a silk fibroin hydrogel (silk 6\%; SPS $10 \mathrm{mM}$ ) performed applying first low strain alternating with high strain values and showing the complete recovery at each cycle; (ii) mesh size ( $\xi$ ) of silk fibroin hydrogels as function of silk fibroin concentration at SPS concentration of $20 \mathrm{mM}$ and (iii) SPS concentration at a silk fibroin concentration of $8 \%$. 
taken as the gelation point; while the point at which the elastic modulus reaches a plateau is considered as the complete crosslinking. Hydrogel precursor solutions with SPS at $40 \mathrm{mM}$ started gelling much faster than solutions containing SPS at 20 $\mathrm{mM}$ and $10 \mathrm{mM}$ (Fig. 2A). Indeed, the gelation point (Fig. 2A(ii)) for SPS $40 \mathrm{mM}$ was recorded at $4.28 \mathrm{~min}( \pm 0.33)$, while SPS $20 \mathrm{mM}$ and $10 \mathrm{mM}$ exhibited a gelation time of $5.15 \mathrm{~min}$ $( \pm 0.55)$ and $6.53 \mathrm{~min}( \pm 0.43)$, respectively. Clearly, a high concentration of SPS ( $40 \mathrm{mM}$ ) led to significantly faster gelation compared to SPS at $10 \mathrm{mM}$, with $10 \mathrm{mM}$ being significantly slower than $20 \mathrm{mM}$ of SPS. Although silk fibroin networks were formed already within $7 \mathrm{~min}$, the in situ crosslinking was monitored for $25 \mathrm{~min}$. The gelation time was directly proportional to the concentration of SPS and the content of persulfate did not affect the elastic modulus once that the reaction was completed, as evidenced by the absence of significant differences in $G^{\prime}$ of samples prepared with different concentrations of SPS (Fig. 2A(iii)). The exposure of RB to visible light produces a photoexcited $\mathrm{RB}$ state, able to abstract electrons from tyrosine residues forming tyrosyl radicals and semireduced $\mathrm{RB}$ radicals. ${ }^{29}$ The coupling of two nearby tyrosyl radicals via carbon-carbon bonds results in di-tyrosine crosslinks. The photo-crosslinking of silk fibroin mediated by RB, can take up to one hour to reach a complete crosslinking resulting in thin films. ${ }^{7}$ We hypothesized that the combination of RB with SPS accelerates the reaction kinetics through continuous regeneration of the excited single state of RB. Further, previous studies showed that the gelation time of keratin-based hydrogels can be decreased from $20 \mathrm{~min}$ to $5 \mathrm{~min}$, increasing the concentration of SPS from $70 \mathrm{mM}$ to $160 \mathrm{mM}^{30}$

Light-induced crosslinking of protein-based materials is likely to involve several reaction pathways due to the presence of amino acids with reactive side chains including tyrosine, tryptophan, lysine, histidine, arginine, methionine and cysteine. ${ }^{29}$ However, most of these amino acids, except tyrosine and lysine, are not present in the silk fibroin chains and lysine accounts only for $0.2 \%$ of the protein. Recently, a study investigating the functional groups involved in the RB-mediated crosslinking, showed a minimal role of primary amines in the network formation. ${ }^{31}$ Therefore, RB induces the formation of the macromolecular network mainly due to the formation of di-tyrosine crosslinks.

We then investigated the effect of silk fibroin concentrations, while keeping SPS and RB at $20 \mathrm{mM}$ and $2 \mathrm{mM}$, respectively (Fig. 2B(i)). Low silk fibroin concentrations (6\% and $8 \%(\mathrm{w} / \mathrm{v})$ ) showed a much faster gelation time compared to solutions with a higher silk content $(10 \%(\mathrm{v} / \mathrm{w}))$ with solutions with a silk fibroin content of $6 \%$ starting gelling at 4.4 minutes $( \pm 0.7)$ while solutions with silk fibroin at $8 \%$ displayed a gelation time of 5.15 minutes $( \pm 0.54)$ (Fig. $2 \mathrm{~B}(\mathrm{ii})$ ). The gelation of silk fibroin solutions at $10 \%$ took much longer. These started to crosslink after 6.68 minutes $( \pm 0.33)$, which was significantly slower than silk fibroin at $6 \%$ and $8 \%$. This might be due to the increased network density that makes the diffusion of the $\mathrm{RB}$ radicals slower and the tyrosine moieties might be not immediately accessible for crosslinking. While the SPS concentration did not affect the elastic modulus of the different compositions once the crosslinking was completed, the silk fibroin content had an influence on the final elastic modulus with silk hydrogels at $6 \%$ showing significantly higher elasticity than at $8 \%$ (Fig. 2B(iii)). Overall, we demonstrated that SPS content can be used to tune the gelation time of silk fibroin hydrogels, whereas the silk fibroin content allows for the modulation of the mechanical properties.

Silk fibroin hydrogels can be fabricated by inducing conformational changes within the silk chains, leading to tough materials with low elasticity; however, the photo-crosslinking, as previously described, ${ }^{20}$ might lower the formation of crystalline regions within the silk chains resulting in materials with higher elasticity. To test the elastic behaviour, the developed silk fibroin hydrogels were subjected to cyclic oscillation tests by alternating low/high shear strain (Fig. 2C(i)). At low shear, the hydrogels exhibited a typical gel behaviour with the elastic modulus higher than the viscous one $\left(G^{\prime}>G^{\prime \prime}\right)$; when increasing the shear strain, the hydrogels displayed a higher viscous modulus $\left(G^{\prime \prime}>G^{\prime}\right)$ showing a typical viscous behaviour. In the next cycles, the hydrogels displayed again a gel property at low shear and this behaviour was reversible during the cycles, showing the excellent elasticity of the silk fibroin hydrogels. The average mesh sizes of the hydrogels were calculated using the rubber elasticity theory and varied between $11 \mathrm{~nm}$ and $14 \mathrm{~nm}$, with silk fibroin at 6\% (w/v) showing the lowest mesh size (Fig. 2C(ii)). Hydrogels with a lower silk fibroin concentration exhibited lower mesh size than samples with higher silk fibroin content, which correlates with the calculated crosslink density (Table S1, ESI $\dagger$ ). In general, the hydrogel's mesh size decreases with increased polymer concentration. However, in our studies it seems that high polymer concentration is associated with a decrease in crosslink density and larger mesh size. This might be explained with the reduced chain mobility that might affect the di-tyrosine coupling. ${ }^{6,32}$ The internal structure of silk fibroin hydrogels was also investigated by SEM and the images are shown in Fig. S2 (ESI $\dagger$ ).

The secondary structure of hydrogels with a silk fibroin content from $6 \%$ to $10 \%(\mathrm{w} / \mathrm{v})$ was investigated using FTIR spectroscopy. Generally, the absorption bands at $1640-1655 \mathrm{~cm}^{-1}$ are associated with the random coils, while the bands at $1610-1630 \mathrm{~cm}^{-1}$ are indicative of a $\beta$-sheet conformation. ${ }^{33-35}$ For all samples (Fig. S3, ESI $\dagger$ ), the presence of absorption bands at $1640-1655 \mathrm{~cm}^{-1}$ was observed, which are associated to the random coils. This suggests that the di-tyrosine crosslinks and the silk fibroin content do not promote the formation of $\beta$-sheet crystalline domains. $^{20}$

\subsection{Physical characterization of RB-mediated crosslinking of silk fibroin hydrogels}

Next, we investigated the effect of SPS on the physical properties of silk fibroin hydrogels by measuring their swelling ratio. The swelling ratio is a measure of the amount of water that a hydrogel can uptake and is predominantly affected by the crosslink density, ${ }^{36}$ as crosslinking hinders the mobility of polymer chains and hence leads to a low swelling ratio. 
A

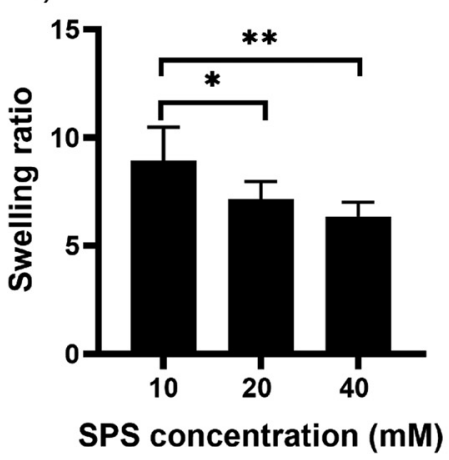

B

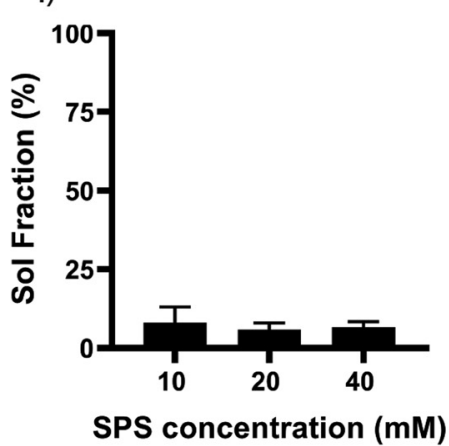

ii)

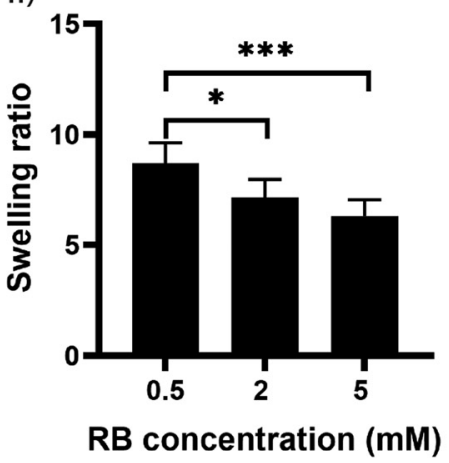

ii)

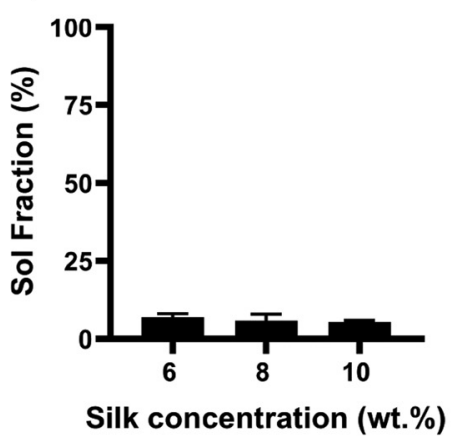

iii)

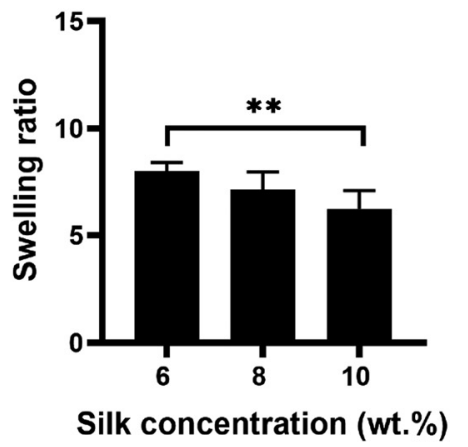

iii)

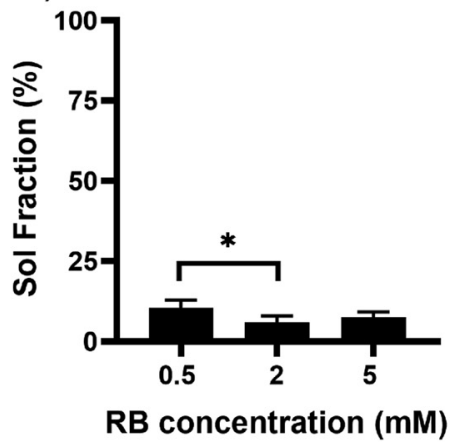

Fig. 3 Swelling ratio (A) and sol fraction (B) of silk fibroin hydrogels prepared by varying (i) SPS concentration while keeping silk fibroin content at $8 \%(\mathrm{w} / \mathrm{v}$ ) and RB at $2 \mathrm{mM}$; (ii) RB concentration with SPS $20 \mathrm{mM}$ and silk fibroin 8\% (w/v); (iii) silk fibroin content with RB $2 \mathrm{mM}$ and SPS $20 \mathrm{mM}$. Data are presented as mean $(n=3-4)$ with standard deviation as error bars (statistical analysis: one-way ANOVA with Tukey's post hoc analysis, ${ }^{\star} p<0.05$, $\left.{ }^{* *} p<0.01,{ }^{* * *} p<0.001\right)$.

Other factors that may also affect the swelling ratio include the presence of hydrophilic or hydrophobic groups, and environmental factors, such as $\mathrm{pH}$, ionic strength, and temperature. ${ }^{37}$ To study the effect of SPS concentration, hydrogels were prepared by maintaining the concentration of $\mathrm{RB}$ and silk fibroin to $2 \mathrm{mM}$ and $8 \%$, respectively. The hydrogels were exposed to visible light for $25 \mathrm{~min}$ and the swelling was determined after soaking the samples at $37{ }^{\circ} \mathrm{C}$ for $24 \mathrm{~h}$ in water (Fig. 3A). The swelling ratio was significantly higher for samples crosslinked with SPS at $10 \mathrm{mM}$, compared to samples that had SPS at $20 \mathrm{mM}$ or $40 \mathrm{mM}$. There was no significant difference between samples crosslinked with $20 \mathrm{mM}$ or $40 \mathrm{mM}$ SPS (Fig. 3A(i)). To evaluate the potential effect on swelling ratio of $\mathrm{RB}$ concentration, we fixed SPS at $20 \mathrm{mM}$ and varied the concentration of RB from $0.5 \mathrm{mM}$ to $5 \mathrm{mM}$ (Fig. 3A(ii)). The swelling ratio decreased by increasing the concentration of $\mathrm{RB}$ from 0.5 to $5 \mathrm{mM}$, with $0.5 \mathrm{mM}$ being significantly different than 2 and $5 \mathrm{mM}$. This suggests that RB at 2 or $5 \mathrm{mM}$ enables a more efficient formation of di-tyrosine crosslinks within the silk chains. Finally, we evaluated the effect of three different silk fibroin concentrations by using SPS $20 \mathrm{mM}$ and RB $2 \mathrm{mM}$ (Fig. 3A(iii)). The swelling ratio was significantly different only between samples having a silk fibroin content of $6 \%$ and $10 \%$. Although $6 \%$ silk fibroin hydrogels exhibited a higher crosslink density, they also showed the highest swelling ratio. Previous studies showed that the formation of inter- and intramolecular interactions within silk molecules, including hydrogen bonds and hydrophobic interactions, increases with higher silk fibroin content, increasing inherent stiffness. ${ }^{38,39}$ This might explain the higher swelling ratio of $6 \%$ hydrogels compared to the $10 \%$ composition. To understand whether the differences in swelling ratio were due to the effect of SPS or RB on the crosslinking efficiency, we performed sol fraction experiments (Fig. 3B). The sol fraction is a measure of the mass of polymer that is not crosslinked in the network. Sol fraction was investigated by varying (i) SPS concentration; (ii) silk fibroin concentration; (iii) RB concentration. All the compositions had a sol-fraction below $10 \%$ and no significant differences were observed, except for RB at $0.5 \mathrm{mM}$, for which the sol fraction was significantly higher than at $2 \mathrm{mM}$. This result indicates that the RB concentration plays a key role in the network formation, whereas SPS and silk fibroin concentrations within the ranges tested do not affect the crosslinking efficiency.

\subsection{Cytocompatibility of RB and SPS}

To assess the effect of the photo-initiator system on the viability of ACPCs, we first tested the effect of the individual components on cells without exposure to visible light (Fig. 4 and 5). At all concentrations tested, RB did not affect cells' viability (Fig. 4), as viability was always higher than $90 \%$. Further, after 


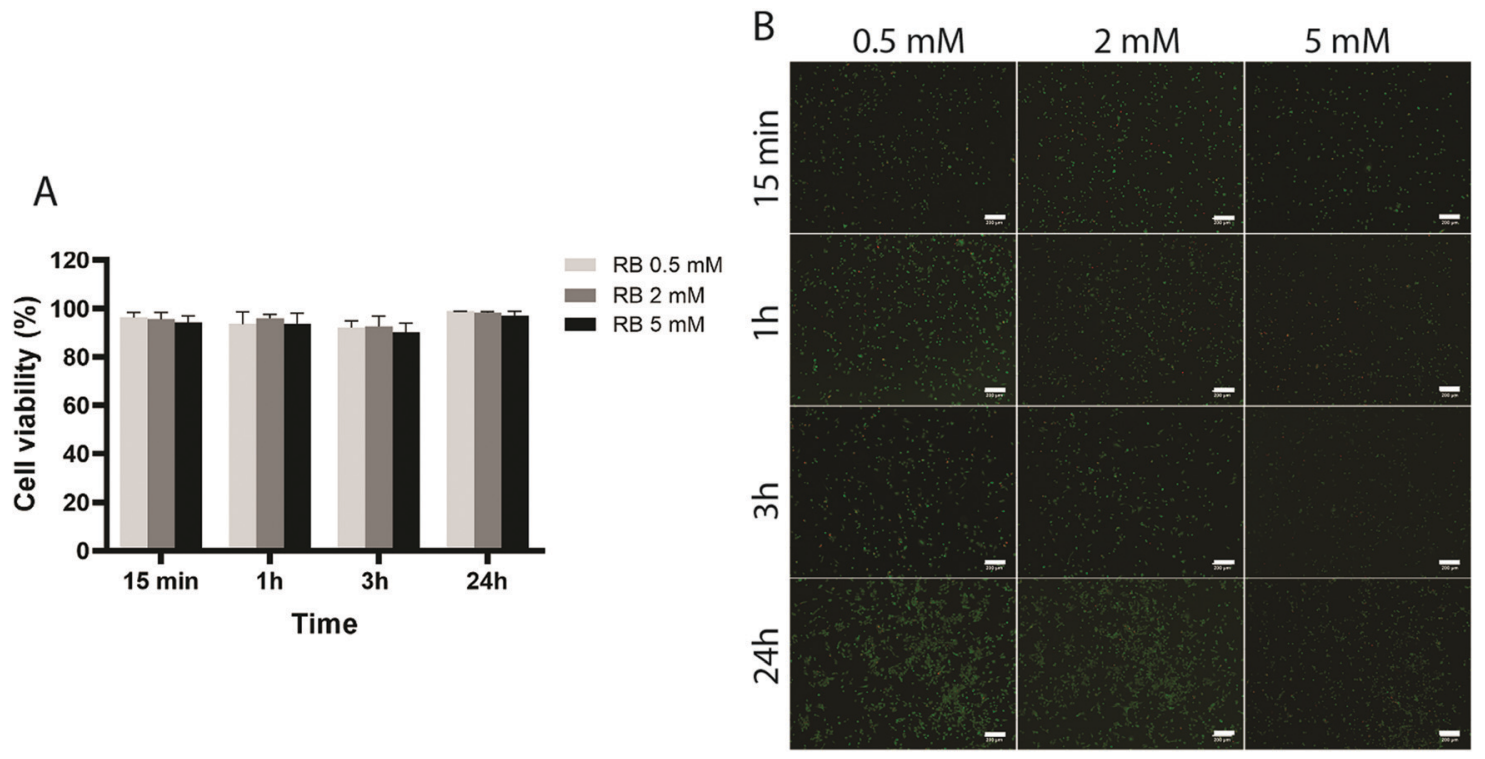

Fig. 4 Effect of RB photo-initiator concentrations on the viability of ACPCs in absence of visible light. (A) Cell viability was determined from the number of live and dead cells obtained by ImageJ analysis $(n=3)$. (B) Fluorescent microscope images (scale bar $200 \mu \mathrm{m}$ ) of live/dead staining of ACPCs after exposure to varying concentration of RB. Data are presented as mean with standard deviation as error bars (statistical analysis: two-way ANOVA with Tukey's post hoc analysis, $\left.{ }^{\star * *} p<0.001\right)$.

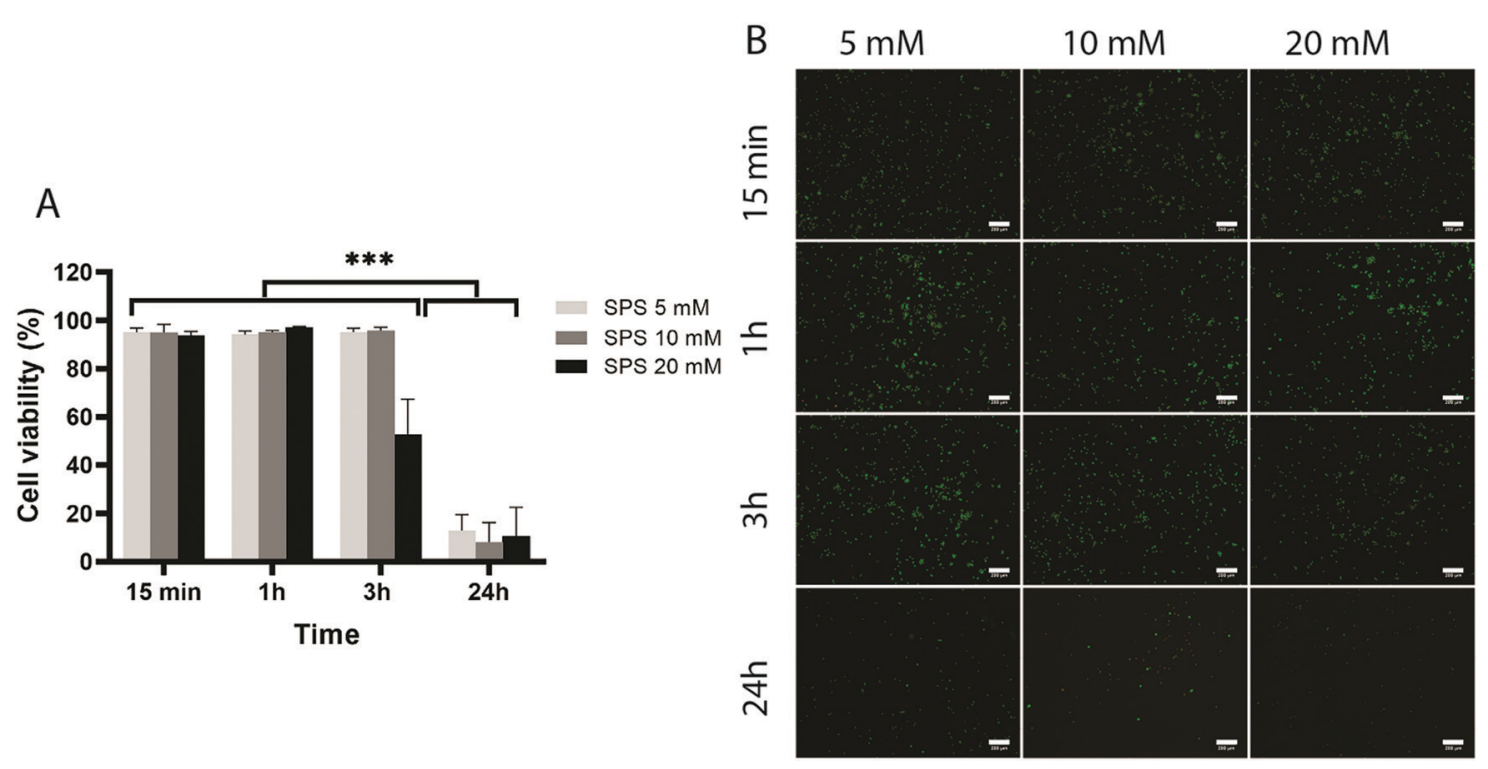

Fig. 5 Effect of SPS concentrations on the viability of ACPCs in absence of visible light. (A) Cell viability was determined from the number of live and dead cells obtained by ImageJ analysis $(n=3)$. (B) Fluorescent microscope images (scale bar $200 \mu \mathrm{m}$ ) of live/dead staining of ACPCs after exposure to varying concentration of SPS. Data are presented as mean with standard deviation as error bars (statistical analysis: two-way ANOVA with Tukey's post hoc analysis, $\left.{ }^{* *} p<0.001\right)$.

24 hours a higher number of cells compared to the start of the experiment was observed, indicating that RB does not impact on cell viability.

Next, we investigated the effect of 5,10 , and $20 \mathrm{mM}$ SPS on cell viability for up to 24 hours (Fig. 5). The direct exposure of SPS to cells, did not had any detectable effect on cell viability in the first hour for the concentrations investigated. However, after 3 hours, cell viability decreased to $52 \%$ for cells exposed to SPS $20 \mathrm{mM}$ and after 24 hours all the concentrations used appeared to be cytotoxic. These results suggest that cell-laden hydrogels can be prepared using SPS at $20 \mathrm{mM}$ if the direct exposure is shorter than 1 hour.

\subsection{Cytocompatibility of cell-laden silk fibroin hydrogels via RB-mediated crosslinking}

Next, we evaluated the cytocompatibility of cell-laden silk fibroin hydrogels via RB-mediated crosslinking. Cytocompatibility was assessed using three cell types of interest to tissue 
A

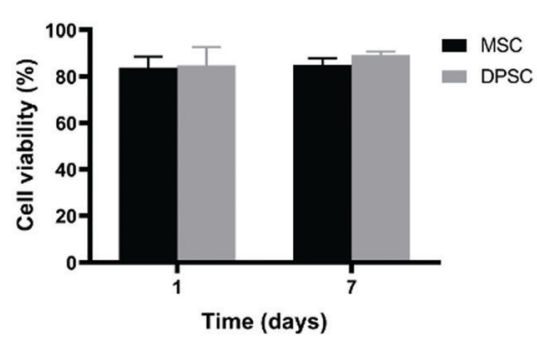

B
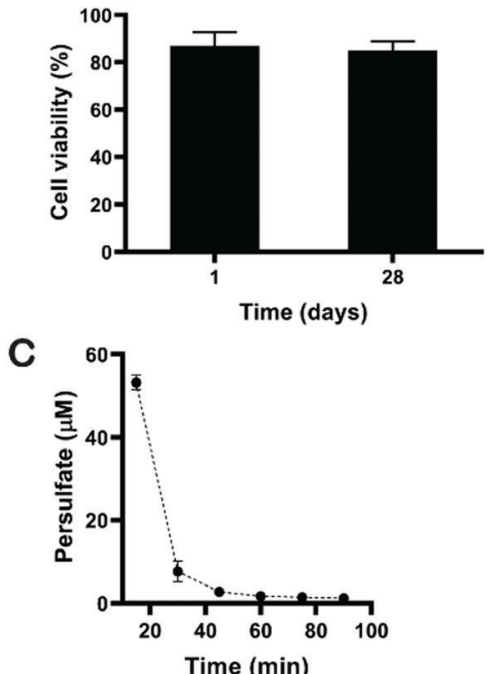
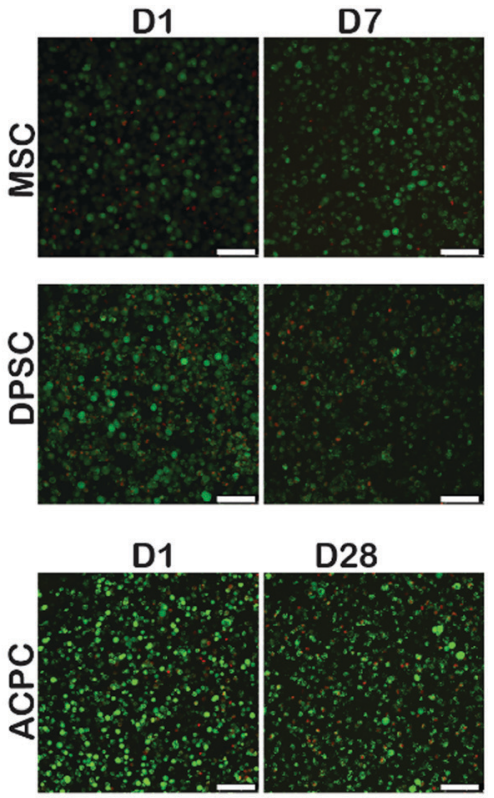

D28

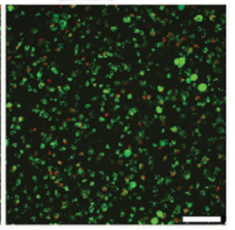

Fig. 6 Viability of cell-laden silk fibroin hydrogels. Silk fibroin hydrogels were prepared by using silk at 6\% (w/v), RB 2 mM, SPS $20 \mathrm{mM}$ and exposure to visible light $10 \mathrm{~min}$. (A) Cell viability and confocal microscopy of live/dead staining of MSC and DPSC encapsulated in silk hydrogels and cultured for 7 days, showing live cells in green and dead cells in red. Scale bars are $100 \mu \mathrm{m}$. (B) Cell viability and confocal microscopy of live/dead staining of silk fibroin hydrogels encapsulating ACPCs and cultured in chondrogenic medium for 28 days (scale bars are $100 \mu \mathrm{m}$ ). Quantification of cell viability (in A and B) was determined from the number of live and dead cells obtained by ImageJ analysis $(n=3)$. (C) Quantification of SPS released from the hydrogels after synthesis, measured using a peroxide assay.

engineering applications, namely MSCs, DPSCs, and ACPCs. ACPCs, like MSCs, are capable of in vitro self-renewal, ${ }^{28,40}$ and produced a higher amount of neo-cartilage matrix when embedded within GelMA hydrogels as compared to expanded chondrocytes. ${ }^{28}$ The DPSCs have received increasing attention, because they have a similar differentiation capability as other MSCs and they are easy to obtain. ${ }^{41}$ These cells can be differentiated into chondrogenic cells and might, thus, be an attractive cell source to replace chondrocytes in cartilage regenerative strategies. ${ }^{42}$

Cells were dispersed within the polymer solution at $15 \times 10^{6}$ cells per $\mathrm{mL}$ and crosslinked using $2 \mathrm{mM}$ of RB, $20 \mathrm{mM}$ of SPS, and $6 \%$ of silk fibroin via $10 \mathrm{~min}$ exposure to visible light (Fig. 6). The swelling behaviour and the shear elastic modulus of silk fibroin hydrogels prepared with an exposure time of $10 \mathrm{~min}$ are shown in Fig. S1 (ESI $\dagger$ ). After crosslinking, the hydrogels were rinsed with growth cell culture medium several times to make sure that any left amount of SPS was washed out, ensuring exposure time remained well below the critical value as shown above. Although, previous studies have shown SPS to be cytotoxic to fibroblast L929 at concentrations above $0.3 \mathrm{mM}$ after 24 hours of exposure, the SPS can be rapidly consumed during the photo-chemical reaction resulting in concentrations below the cytotoxic threshold. ${ }^{43}$ To confirm that SPS could be effectively removed from hydrogels via washing steps, the amount of SPS released from the hydrogels was measured using a peroxide assay. After $15 \mathrm{~min}$, the amount of SPS was below $60 \mu \mathrm{M}$, which is well below the determined cytotoxic limit (Fig. 6C). The hydrogels encapsulating MSCs and DPSCs were cultured for 7 days. Silk hydrogels encapsulating ACPCs were incubated in chondrogenic medium and cultured for a longer period of 28 days to assess the cytocompatibility of the visible light induced RB crosslinking. To evaluate the viability of encapsulated cells, live/dead assays were performed at the beginning and end of the culture periods, i.e. at 1 day and 7 days for MSCs and DPSCs, and at 1 day and 28 days for ACPCs. At all time-points, the viability was above $80 \%$ for all cell types, which was substantially higher than those reported for human articular chondrocytes encapsulated in silk hydrogels that were prepared by ruthenium/SPS photo-crosslinking. ${ }^{20}$ 
These results demonstrate that RB-mediated crosslinking supports the viability of different cell types following encapsulation and prolonged in vitro culture.

\section{Conclusions}

In this study, we demonstrated a novel approach for the encapsulation of mammalian cells in rapid crosslinking silk hydrogels fabricated using a non-toxic photo-initiator. Although the use of $\mathrm{RB}$ has been previously described for the photo-crosslinking of silk, this is the first study in which cells were encapsulated within the hydrogel matrix and present during the photo-chemical reaction. The approach investigated here enables the crosslinking of silk hydrogel via formation of di-tyrosine crosslinks. The reaction kinetics can be modulated by varying the content of the electron acceptor in combination with $\mathrm{RB}$, whereas the mechanical properties are mainly controlled by the polymer content. Further, we demonstrated that the hydrogel formation rate can be controlled by the amount of SPS and the final storage modulus does not depend on SPS concentration. Silk hydrogels prepared by combining RB with SPS and visible light (600 lumen white lamp) can support the viability of different mammalian cell types, and could potentially act as a promising bioink formulation for bioprinting application. Future studies will focus on the investigation of the chondrogenic potential of this hydrogel formulation.

\section{Conflicts of interest}

There are no conflicts to declare.

\section{Acknowledgements}

The authors would like to thank Riccardo Levato and Lizette Utomo for kindly providing ACPCs and DPSCs, respectively. The authors also thank Thomas N. Ran for the support with FTIR characterization. This work was supported by the Dutch Arthritis Association (LLP12, LLP22, and LLP25); and the strategic alliance program entitled: Advanced Biomanufacturing, funded by the University of Twente, Utrecht University and University Medical Center Utrecht, project title: Bioprinting functional tissues from stem cells and enabling biomaterials.

\section{References}

1 M. W. Tibbitt and K. S. Anseth, Biotechnol. Bioeng., 2009, 103, 655-663.

2 D. Huang, Y. Huang, Y. Xiao, X. Yang, H. Lin, G. Feng, X. Zhu and X. Zhang, Acta Biomater., 2019, 97, 74-92.

3 M. Guvendiren and J. A. Burdick, Curr. Opin. Biotechnol, 2013, 24, 841-846.

4 B. V. Slaughter, S. S. Khurshid, O. Z. Fisher, A. Khademhosseini and N. A. Peppas, Adv. Mater., 2009, 21, 3307-3329.

5 A. M. Jonker, D. W. Löwik and J. C. van Hest, Chem. Mater., 2012, 24, 759-773.
6 O. Hasturk, K. E. Jordan, J. Choi and D. L. Kaplan, Biomaterials, 2020, 232, 119720.

7 M. B. Applegate, B. P. Partlow, J. Coburn, B. Marelli, C. Pirie, R. Pineda, D. L. Kaplan and F. G. Omenetto, Adv. Mater., 2016, 28, 2417-2420.

8 D. G. Harkin, K. A. George, P. W. Madden, I. R. Schwab, D. W. Hutmacher and T. V. Chirila, Biomaterials, 2011, 32, 2445-2458.

9 D. Chouhan, T. u. Lohe, P. K. Samudrala and B. B. Mandal, Adv. Healthcare Mater., 2018, 7, 1801092.

10 W. Shi, M. Sun, X. Hu, B. Ren, J. Cheng, C. Li, X. Duan, X. Fu, J. Zhang and H. Chen, Adv. Mater., 2017, 29, 1701089.

11 S. Kapoor and S. C. Kundu, Acta Biomater., 2016, 31, 17-32.

12 C. Holland, K. Numata, J. Rnjak-Kovacina and F. P. Seib, Adv. Healthcare Mater., 2019, 8, 1800465.

13 W. H. Elliott, W. Bonani, D. Maniglio, A. Motta, W. Tan and C. Migliaresi, ACS Appl. Mater. Interfaces, 2015, 7, 12099-12108.

14 M. Lee, S. Seo and J. C. Kim, Clin. Exp. Dermatol., 2012, 37, 762-771.

15 S. Das, F. Pati, Y.-J. Choi, G. Rijal, J.-H. Shim, S. W. Kim, A. R. Ray, D.-W. Cho and S. Ghosh, Acta Biomater., 2015, 11, 233-246.

16 B. P. Partlow, C. W. Hanna, J. Rnjak-Kovacina, J. E. Moreau, M. B. Applegate, K. A. Burke, B. Marelli, A. N. Mitropoulos, F. G. Omenetto and D. L. Kaplan, Adv. Funct. Mater., 2014, 24, 4615-4624.

17 F. Lee, K. H. Bae and M. Kurisawa, Biomed. Mater., 2015, 11, 014101.

18 D. A. Fancy and T. Kodadek, Proc. Natl. Acad. Sci. U. S. A., 1999, 96, 6020-6024.

19 J. L. Whittaker, N. R. Choudhury, N. K. Dutta and A. Zannettino, J. Mater. Chem. B, 2014, 2, 6259-6270.

20 X. Cui, B. G. Soliman, C. R. Alcala-Orozco, J. Li, M. A. Vis, M. Santos, S. G. Wise, R. Levato, J. Malda and T. B. Woodfield, Adv. Healthcare Mater., 2020, 9(4), 1901667.

21 W. Han Ang and P. J. Dyson, Eur. J. Inorg. Chem., 2006, 4003-4018.

22 O. Novakova, J. Kasparkova, O. Vrana, P. M. van Vliet, J. Reedijk and V. Brabec, Biochemistry, 1995, 34, 12369-12378.

23 H. Y. Liu, H. D. Nguyen and C. C. Lin, Adv. Healthcare Mater., 2018, 7, 1800954.

24 C. P. Camp, I. L. Peterson, D. S. Knoff, L. G. Melcher, C. J. Maxwell, A. T. Cohen, A. M. Wertheimer and M. Kim, Front. Chem., 2020, 8, 173.

25 D. N. Rockwood, R. C. Preda, T. Yücel, X. Wang, M. L. Lovett and D. L. Kaplan, Nat. Protoc., 2011, 6, 1612.

26 N. Lohmann, L. Schirmer, P. Atallah, E. Wandel, R. A. Ferrer, C. Werner, J. C. Simon, S. Franz and U. Freudenberg, Sci. Transl. Med., 2017, 9, eaai9044.

27 J. Karvinen, T. O. Ihalainen, M. T. Calejo, I. Jönkkäri and M. Kellomäki, Mater. Sci. Eng., C, 2019, 94, 1056-1066.

28 R. Levato, W. R. Webb, I. A. Otto, A. Mensinga, Y. Zhang, M. van Rijen, R. van Weeren, I. M. Khan and J. Malda, Acta Biomater., 2017, 61, 41-53. 
29 R. W. Redmond and I. E. Kochevar, Photochem. Photobiol., 2019, 95, 1097-1115.

30 J. K. Placone, J. Navarro, G. W. Laslo, M. J. Lerman, A. R. Gabard, G. J. Herendeen, E. E. Falco, S. Tomblyn, L. Burnett and J. P. Fisher, Ann. Biomed. Eng., 2017, 45, 237-248.

31 A. S. McCall, S. Kraft, H. F. Edelhauser, G. W. Kidder, R. R. Lundquist, H. E. Bradshaw, Z. Dedeic, M. J. Dionne, E. M. Clement and G. W. Conrad, Invest. Ophthalmol. Visual Sci., 2010, 51, 129-138.

32 M. McGill, J. M. Coburn, B. P. Partlow, X. Mu and D. L. Kaplan, Acta Biomater., 2017, 63, 76-84.

33 D. Su, M. Yao, J. Liu, Y. Zhong, X. Chen and Z. Shao, ACS Appl. Mater. Interfaces, 2017, 9, 17489-17498.

34 J. Choi, M. McGill, N. R. Raia, O. Hasturk and D. L. Kaplan, Adv. Healthcare Mater., 2019, 8, 1900644.

35 X. Wang, Z. Ding, C. Wang, X. Chen, H. Xu, Q. Lu and D. L. Kaplan, J. Mater. Chem. B, 2018, 6, 2739-2746.
36 N. A. Peppas, J. Z. Hilt, A. Khademhosseini and R. Langer, Adv. Mater., 2006, 18, 1345-1360.

37 N. Peppas, P. Bures, W. Leobandung and H. Ichikawa, Eur. J. Pharm. Biopharm., 2000, 50, 27-46.

38 U.-J. Kim, J. Park, C. Li, H.-J. Jin, R. Valluzzi and D. L. Kaplan, Biomacromolecules, 2004, 5, 786-792.

39 G. D. Kang, J. H. Nahm, J. S. Park, J. Y. Moon, C. S. Cho and J. H. Yeo, Macromol. Rapid Commun., 2000, 21, 788-791.

40 H. E. McCarthy, J. J. Bara, K. Brakspear, S. K. Singhrao and C. W. Archer, Vet. J., 2012, 192, 345-351.

41 A. Longoni, L. Utomo, I. van Hooijdonk, G. Bittermann, V. Vetter, E. K. Spanjer, J. Ross, A. Rosenberg and D. Gawlitta, Eur. Cells Mater., 2020, 39, 121.

42 L. Yao and N. Flynn, Spine J., 2018, 18, 1070-1080.

43 C. M. Elvin, T. Vuocolo, A. G. Brownlee, L. Sando, M. G. Huson, N. E. Liyou, P. R. Stockwell, R. E. Lyons, M. Kim and G. A. Edwards, Biomaterials, 2010, 31, 8323-8331. 